\title{
August Issue of Stem Cell Reviews and Reports Including a Spotlight Paper from Dr. Hal Broxmeyer on a Role of Brain in Regulating Hematopoiesis
}

\author{
Mariusz Z. Ratajczak ${ }^{1}$ \\ Published online: 15 July 2021 \\ (c) The Author(s), under exclusive licence to Springer Science+Business Media, LLC, part of Springer Nature 2021
}

In this August issue of Stem Cell Reviews and Reports, we publish several exciting reviews and original papers on different types of stem cells. Our journal is open to scientific discussions, sometimes even controversial, and to presenting novel data. Our scientific mottos are famous sayings from Albert Einstein that "Blind believe in the authority is the greatest enemy of truth" and from Max Planck, "One rule is important in science-only courageous people win". Thus, we are open to present scientific progress in the field and new challenging ideas and discuss some controversies in the field. We are still looking for a stem cell that could be safely and efficiently employed in regenerative medicine.

Beginning with this issue of Stem Cell Reviews and Reports, we will also publish spotlight papers submitted by leading investigators in the field. In the current issue, we present an excellent and visionary spotlight paper from Dr. Hal Broxmeyer 's group on the role of the brain in hematopoiesis [1]. The nervous system and hemato/lymphopoiesis are crucial for organism survival and adaptation to the environment and, for many years, were thought to act independently. However, as nicely discussed and summarized in this August issue, spotlight paper evidence demonstrates an active and beneficial dialogue between the central nervous system and hemato/lymphopoiesis. Therefore, a new growing field in the life sciences emerges that deals with neuronal regulation of the production of blood cells.

One can ask how the central nervous system regulates the directly or indirectly production of blood elements. To understand this, the role of the brain is to integrate signals both from the surrounding environment and collected from the organs and tissues and translate into responses that allow survival and adaptation to detected changes. One of

Mariusz Z. Ratajczak

mariusz.ratajczak@louisville.edu

1 University of Louisville, Louisville, KY, USA the potent synchronizing factors is inner clock located in the suprachiasmatic nucleus in hypothalamus, responsible for circadian rhythm. It has been nicely demonstrated that circadian rhythm regulates both production of blood cells, their circulation in peripheral blood as well affects immune responses [2]. Besides, circadian inner clock, the major brain initiated pathways that regulate hemato/lymphopoiesis involve i) endocrine signals generated from pituitary and pineal gland that directly or indirectly via peripheral endocrine glands affect hematopoietic organs, ii) the neurological pathways involving adrenergic and cholinergic neurological signaling arms modulated at the synaptic and postsynaptic level by purinergic signaling mediators, iii) meningeal lymphatics and iv) peripheral sensory pathways. The effects of the central nervous system on hematopoiesis have been demonstrated in steady-state conditions and in patients suffering from brain damage, e.g., as seen in patients after stroke [3].

To address this better pituitary gland that is an anatomical part of the brain may directly affect hematopoiesis and, as recently demonstrated, both follicle-stimulating factor (FSH) and luteinizing hormone (LH) directly promote proliferation of hemato/lymphopoietic cells [4]. Moreover, by stimulating adrenal and thyroid glands, the pituitary gland promotes additionally formation of blood and immune cells. In addition, pineal gland-derived melatonin is an essential modulator of circadian rhythm and stimulator of hemato/ lymphopoiesis [2]. Furthermore, peripheral hematopoietic organs, including bone marrow, are innervated by neural fibers, and both adrenergic and cholinergic mediators affect hematopoiesis. Mounting evidence accumulated that both norepinephrine and acetylcholine regulate the specification and trafficking of hematopoietic cells [5]. What is also important to consider extracellular adenosine triphosphate (eATP), acting as a signaling molecule in the purinergic network, stored in vesicles at the synaptic endings, and released after neuronal depolarization together with several other neurotransmitters such as acetylcholine or norepinephrine. 
Purinergic signaling mediators that are involved in brain functioning are known modulators of hematopoiesis and hematopoietic stem cell trafficking [4].

Based on the aforementioned, we encourage readers to follow Dr. Broxmeyer's visionary paper and to study a role of the central nervous system in regulating the development of hematopoietic cells. In stem cell biology, in order to understand this fascinating cell system, there are still many doors to be opened for investigations, and one of such directions is very nicely presented in this currently published excellent spotlight.

\section{References}

1. Broxmeyer, H. E., Yoder, K. K., Wu, Y. Ch., Hutchins, G. D., Cooper, S. H., \& Farag, S. S. (2021). The brain: Is it a next frontier to better understand the regulation and control of hematopoiesis for future modulation and treatment? Stem Cell Reviews and Reports. https://doi.org/10.1007/s12015-021-10203-0
2. Golan, K., Kumari, A., Kollet, O., Khatib-Massalha, E., Subramaniam, M. D., Ferreira, Z. S., et al. (2018). Daily onset of light and darkness differentially controls hematopoietic stem cell differentiation and maintenance. Cell Stem Cell, 23, 572-585.e7.

3. Courties, G., Herisson, F., Sager, H. B., Heidt, T., Ye, Y., Wei, Y., et al. (2015). Ischemic stroke activates hematopoietic bone marrow stem cells. Circulation Research, 116, 407-417.

4. Bujko, K., Cymer, M., Adamiak, M., \& Ratajczak, M. Z. (2019). An overview of novel unconventional mechanisms of hematopoietic development and regulators of hematopoiesis - A roadmap for future investigations. Stem Cell Reviews and Reports, 15, 785-794.

5. Méndez-Ferrer, Simón, Battista, Michela, \& Frenette, Paul S. (2010). Cooperation of $\beta 2$ - and $\beta 3$-adrenergic receptors in hematopoietic progenitor cell mobilization: $\beta$-adrenoceptors in bone marrow microenvironment. Annals of the New York Academy of Sciences, 1192(1), 139-144. https://doi.org/10.1111/j.1749-6632. 2010.05390.x

Publisher's Note Springer Nature remains neutral with regard to jurisdictional claims in published maps and institutional affiliations. 